\title{
On Determinants of Laplacians on Riemann Surfaces
}

\author{
Eric D’Hoker ${ }^{\star}$ and D. H. Phong ${ }^{\star \star}$ \\ Departments of Physics and Mathematics, Columbia University, New York, NY 10027, USA
}

\begin{abstract}
Determinants of Laplacians on tensors and spinors of arbitrary weights on compact hyperbolic Riemann surfaces are computed in terms of values of Selberg zeta functions at half integer points.
\end{abstract}

\section{Introduction}

In this paper we evaluate explicitly the determinants of Laplacians acting on arbitrary tensor and spinor fields on compact Riemann surfaces of constant negative curvature. They are equal to values of Selberg zeta functions at halfinteger points, multiplied by an additional factor depending only on the genus and the weight of the field. Interest in such results comes from multiloop calculations for fermionic string theories and random surfaces, where these determinants arise from quantum fluctuations and Faddeev-Popov gauge fixing, while the extra factors can be viewed as finite corrections to the coupling constants [1].

Our approach is based on the explicit formulas for heat kernels of Fay [2], the Maass operators, and Selberg trace formulas. We observe that Selberg trace formulas have been used in similar contexts by many authors, notably Ray and Singer [3(a)], Donnelly [3(b)], McKean [4], Hejhal [5], Mandelstam [6], and Fried [7].

\section{Tensors, Spinors, and Automorphic Forms}

Let $M$ be a compact Riemann surface with a fixed hermitian metric $d s^{2}$ of constant curvature $-1, \chi=2-2 h$ its Euler characteristic $(\chi<0)$, and let $T^{n}$ denote the usual space of tensors $\left\{f(z) d z^{n}\right\}$ for $n$ integer. If we fix a spinor structure among the $2^{2 h}$ possible ones, we may also consider $n=\left(\right.$ odd integer) $/ 2$, and view $T^{1 / 2}$ as the space of spinors, and $T^{n}$ as spaces of spinor-tensor fields. Henceforth $n$ will be allowed to take both integer and half-integer values. The covariant derivative $\nabla$ sends $T^{n}$ into

* Research supported in part by the U.S. Department of Energy

$\star \star$ Research supported in part by the National Science Foundation under Grant DMS-84-02710 
$T^{n} \otimes\left(T^{1} \oplus \bar{T}^{1}\right)$, and can be decomposed accordingly as $\nabla=\nabla_{z}^{n} \oplus \nabla_{n}^{z}$, with $\nabla_{z}^{n}: T^{n}$ $\rightarrow T^{n} \otimes T^{1} \simeq T^{n+1}, \nabla_{n}^{z}: T^{n} \rightarrow T^{n} \otimes \bar{T}^{1} \simeq T^{n-1}$. The spaces $T^{n}$ are Hilbert spaces, since spinor-tensor fields can be paired at each point of $M$, and then integrated over $M$ using $d s^{2}$. With this pairing, $\left(\nabla_{z}^{n}\right)^{+}=-\nabla_{n}^{z}, \nabla_{1 / 2}^{z}$ is the Dirac operator, and the natural covariant Laplacians on $T^{n}$ are

$$
\Delta_{n}^{+}=-\nabla_{n}^{z} \nabla_{z}^{n}, \quad \Delta_{n}^{-}=-\nabla_{z}^{n} \nabla_{n}^{z} .
$$

In local isothermal coordinates $z$, we can write

$$
\begin{gathered}
d s^{2}=2 g_{z \bar{z}} d z d \bar{z}, \quad \nabla_{n}^{z} f=g^{z \bar{z}} \partial_{\bar{z}} f, \\
\nabla_{z}^{n} f=\left(g_{z \bar{z}}\right)^{n} \partial_{z}\left(\left(g^{z \bar{z}}\right)^{n} f\right), \quad\langle f \mid g\rangle_{T^{n}}=\int_{M} d z d \bar{z} g_{z \bar{z}}\left(g^{z \bar{z}}\right)^{n} f^{*} g .
\end{gathered}
$$

The uniformization theorem allows us to identify $M$ with $H / \Gamma$, where $H$ is the upper half plane $\left\{z=x+i y, y>0, d s^{2}=y^{-2} d z d \bar{z}\right\}, \Gamma$ is a discrete subgroup of $\operatorname{SL}(2, \mathbb{R}) /\{ \pm 1\}$, all of whose elements $\left(\begin{array}{ll}a & b \\ c & d\end{array}\right)$ are hyperbolic, i.e., $|a+d|>2$. Let $\tilde{\Gamma}$ be the subgroup of $\operatorname{SL}(2, \mathbb{R})$ containing $-I$ which projects to $\Gamma$, and let $\gamma_{1}, \gamma_{2}, \ldots, \gamma_{2 h}$ be a fixed set of generators for $\tilde{\Gamma}$. A spin structure $v$ on $M$ corresponds to a choice of multipliers $v(\gamma) \in\{ \pm 1\}$ on $\gamma \in \tilde{\Gamma}$ which is multiplicative and satisfies $v(-I)=-1$. Such a choice is determined by the values of $v$ on the generators $\gamma_{i}$, and there are $2^{2 h}$ of them. Once a multiplier $v$ is fixed, $v(\tilde{\gamma})(c z+d)$ is well defined for $\tilde{\gamma} \in \operatorname{SL}(2, \mathbb{R}), \tilde{\gamma}=\left(\begin{array}{ll}a & b \\ c & d\end{array}\right), \tilde{\gamma}$ representative of $\gamma$ in $\operatorname{SL}(2, \mathbb{R}) /\{ \pm 1\}$. For $p, q$ of the type (integer) $/ 4$, satisfying $p+q=$ (integer) $/ 2$, the fields $f(z)(d z)^{p}(d \bar{z})^{q}$ defined globally on $H$ and transforming as

$$
f(\gamma z)=[v(\tilde{\gamma})]^{2(p+q)}(c z+d)^{2 p}(c \bar{z}+d)^{2 q} f(z), \quad \gamma \in \Gamma
$$

project uniquely to fields of the same weight on $H / \Gamma$. They carry a natural inner product

$$
\langle f \mid g\rangle=\int_{H / \Gamma} d z d \bar{z} y^{-2+p+q} f^{*} g .
$$

The fields in $T^{n}$ correspond to the case $n=p$ and $q=0$. The inner product (1.2) on $T^{n}$ corresponds under these conditions to the inner product (1.4). It will actually be more convenient to work with the space

$$
S(n)=\{f \text { satisfying (1.3) with }-q=p=n / 2\},
$$

which is isometric to $T^{n}$ through the correspondence

$$
T^{n} \ni f \stackrel{I}{\longrightarrow} y^{n / 2} f \in S(n) .
$$

Under this correspondence, the operators $\nabla_{n}^{z}, \nabla_{z}^{n}$ go over to the Maass operators $L_{n}: S(n) \rightarrow S(n-1), K_{n}: S(n) \rightarrow S(n+1)$ according to the diagram

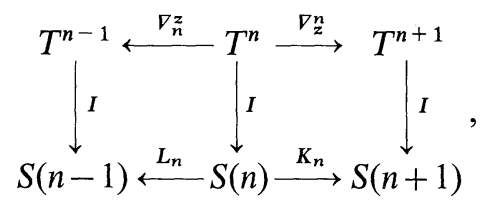


where

$$
L_{n}=(\bar{z}-z) \frac{\partial}{\partial \bar{z}}-n, \quad K_{n}=(z-\bar{z}) \frac{\partial}{\partial z}+n .
$$

In particular the Laplacians $\Delta_{n}^{ \pm}$reduce to

$$
\Delta_{n}^{+}=-L_{n+1} K_{n}=-D_{-n}+n(n+1), \quad \Delta_{n}^{-}=-K_{n-1} L_{n}=-D_{-n}+n(n-1)
$$

with

$$
D_{n}=y^{2}\left(\frac{\partial^{2}}{\partial x^{2}}+\frac{\partial^{2}}{\partial y^{2}}\right)-2 i n y \frac{\partial}{\partial x} \text {. }
$$

\section{Traces of Heat Kernels on Spinors-Tensors of Arbitrary Weights}

This section is devoted to the computation of $\operatorname{Tr}\left(e^{-t \Delta_{n}^{ \pm}}\right)$for $n$ arbitrary halfinteger. ${ }^{1}$ The starting point is the formula for the kernel $g_{n}^{t}\left(z, z^{\prime}\right)$ of $e^{t D_{n}}$ on the upper half plane obtained by Fay [2, p. 157]

$$
\begin{aligned}
g_{n}^{t}\left(z, z^{\prime}\right)= & \sum_{0 \leqq m<|n|-\frac{1}{2}} e^{(|n|-m)(|n|-m-1) t} \Lambda_{n, m}(d) \\
& +\frac{\sqrt{2} e^{-t / 4}}{(4 \pi t)^{3 / 2}} \int_{d}^{\infty} d b \frac{b e^{-b^{2} / 4 t}}{\sqrt{\cosh b-\cosh d}} \\
& \cdot\left\{\frac{1}{2}(\cosh d-1)^{-n}\left[e^{-2 n \theta} A_{+}^{2 n}+e^{2 n \theta} A_{-}^{2 n}\right]\right\},
\end{aligned}
$$

where $d=\operatorname{Arg} \cosh \left(1+\frac{\left|z-z^{\prime}\right|^{2}}{2 y y^{\prime}}\right)$ is the distance between $z$ and $z^{\prime}$;

$$
\begin{aligned}
\Lambda_{n, m}= & \frac{(-1)^{m}}{4 \pi m !} \frac{\Gamma(2|n|-m)}{\Gamma(2|n|-2 m-1)}\left(\frac{2}{1+\cosh d}\right)^{|n|-m} \\
& \cdot F\left(-m, 2|n|-m, 2|n|-2 m ; \frac{2}{1+\cosh d}\right) .
\end{aligned}
$$

$F$ is the usual hypergeometric function,

$$
\begin{aligned}
e^{ \pm \theta} \sinh d & =e^{b}-\cosh d \pm e^{b / 2} \sqrt{2(\cosh b-\cosh d)}, \\
A_{ \pm} & =\sqrt{2} \sinh b / 2 \pm \sqrt{\cosh b-\cosh d} .
\end{aligned}
$$

The integrand on (2.1) can be simplified considerably. If we set

$$
E_{n}(b, d)=2^{-1}(\cosh d-1)^{-n / 2}\left(e^{-n \theta} A_{+}^{n}+e^{n \theta} A_{-}^{n}\right),
$$

and note that $A_{+} A_{-}=(\cosh d-1)$, we see immediately that $E_{n}=E_{-n}$. Next a routine calculation shows that $E_{0}=1, E_{1}=(\cosh b / 2) /(\cosh d / 2)$, and that $2 E_{1} E_{n}$ $=E_{n+1}+E_{n-1}$, which is the defining relation for Chebyshev polynomials. We may

\footnotetext{
${ }^{1}$ Traces are always considered over complex functions, and all dimensions considered throughout are complex dimensions
} 
thus restrict ourselves to $n \geqq 0, n$ half integer, and write

$$
\begin{aligned}
g_{n}^{t}\left(z, z^{\prime}\right)= & \sum_{0 \leqq m<n-\frac{1}{2}} e^{(n-m)(n-m-1) t} \Lambda_{n, m}(d) \\
& +\frac{\sqrt{2} e^{-t / 4}}{(4 \pi t)^{3 / 2}} \int_{d}^{\infty} d b \frac{b e^{-b^{2} / 4 t}}{\sqrt{\cosh b-\cosh d}} T_{2 n}((\cosh b / 2) /(\cosh d / 2)),
\end{aligned}
$$

where $T_{2 n}$ is the $(2 n)^{\text {th }}$ Chebyshev polynomial.

The heat kernel $K_{n}^{t}\left(z, z^{\prime}\right)$ on the Riemann surface $H / \Gamma$ can now be obtained by taking the Poincaré series

$$
K_{n}^{t}\left(z, z^{\prime}\right)=\sum_{\gamma \in \Gamma} v(\gamma)^{2 n}\left(\frac{c z+d}{c \bar{z}+d}\right)^{n}\left(\frac{z-\gamma \bar{z}^{\prime}}{\gamma z^{\prime}-\bar{z}}\right)^{n} g_{n}^{t}\left(z, \gamma z^{\prime}\right)
$$

To compute its trace, we apply Selberg trace formula techniques (described in detail for example in $[4,1 d])$ and obtain

$$
\begin{aligned}
\operatorname{Tr}\left(e^{t D_{n}}\right) & =\int_{H / \Gamma} \frac{d x d y}{y^{2}} g_{n}^{t}(z, z)+\sum_{\gamma \text { primitive }} v(\gamma)^{2 n} \sum_{p=1}^{\infty} \int_{-\infty}^{\infty} d x \int_{1}^{e^{l}} \frac{d y}{y^{2}}\left(\frac{z-e^{p l} \bar{z}}{e^{p l} z-\bar{z}}\right)^{n} g_{n}^{t}\left(z, e^{l p} z\right) \\
& \equiv I_{e}^{n}(t)+I^{n}(t) .
\end{aligned}
$$

Here the sum over $\gamma$ primitive indicates summing over all $\gamma$ 's which are not powers of another element in $\Gamma$ with exponent $\geqq 2$ (if $\gamma$ is primitive, $\gamma^{-1}$ is also counted as primitive), and for each $\gamma$ the corresponding length of a closed geodesic $l$ is given by $\cosh l / 2=|\operatorname{trace} \gamma| / 2$. We have also chosen the representative of $\gamma$ in $\operatorname{SL}(2, \mathbb{R})$ to be with positive trace.

Computation of $I^{n}(t)$. To compute the integrals in (2.4) we change the variables from $z=x+i y$ to $(x, u=x / y)$, set $\alpha=2^{1 / 2} \sinh (p l / 2)$, and note that

$$
d=d\left(z, e^{l p} z\right)=\operatorname{Arg} \cosh \left(\alpha^{2} u^{2}+\cosh p l\right), \quad \frac{z-e^{p l} \bar{z}}{e^{p l} z-\bar{z}}=\frac{\cosh p l / 2+i u \sinh p l / 2}{\cosh p l / 2-i u \sinh p l / 2} .
$$

The expression $I^{n}(t)$ becomes then the sum over $\gamma$ and $p$ of $(v(\gamma))^{2 n}$ times

$$
\begin{aligned}
l & \sum_{0 \leqq m<n-\frac{1}{2}} e^{(n-m)(n-m-1) t} \int_{-\infty}^{\infty} d u\left(\frac{\cosh p l / 2+i u \sinh p l / 2}{\cosh p l / 2-i u \sinh p l / 2}\right)^{n} \Lambda_{n, m}(d) \\
& +l \frac{\sqrt{2} e^{-t / 4}}{(4 \pi t)^{3 / 2}} \int_{-\infty}^{\infty} d u\left(\frac{\cosh p l / 2+i u \sinh p l / 2}{\cosh p l / 2-i u \sinh p l / 2}\right)^{n} \\
& \cdot \int_{d}^{\infty} d b \frac{b e^{-b^{2} / 4 t}}{\sqrt{\cosh b-\cosh d}} T_{2 n}\left(\frac{\cosh b / 2}{\cosh d / 2}\right)
\end{aligned}
$$

Next we observe that $F\left(-m, 2|n|-m, 2|n|-2 m, 2(1+\cosh d)^{-1}\right)$ is a polynomial of degree $\leqq m$ in $(1+\cosh d)^{-1}$, and that

$$
(1+\cosh d)=[\cosh p l / 2+i u \sinh p l / 2][\cosh p l / 2-i u \sinh p l / 2] \text {. }
$$


It follows that the first integral in (2.5) can be treated by a contour integration and shown to vanish for all $n$ half-integers. To compute the remaining integrals we first interchange the $u$ and $b$ integrations and then introduce a parameter $\lambda$ and the generating function for Chebyshev polynomials. With new integration variable $\theta$ given by $u=\omega \sin \theta / \alpha,\left(\omega^{2}=\cosh b-\cosh l p\right) B=\sqrt{2} \cosh (p l / 2) / \omega$, the result is

$$
\begin{aligned}
& \sum_{\substack{n=0 \\
\text { integer }}}^{\infty} \lambda^{n} \int_{-\omega / \alpha}^{\omega / \alpha} d u\left(\frac{\cosh p l / 2+i u \sinh p l / 2}{\cosh p l / 2-i u \sinh p l / 2}\right)^{n / 2} T_{n}\left(\frac{\cosh b / 2}{\cosh d / 2}\right) \\
& \quad=\sum_{\substack{n=0 \\
\text { integer }}}^{\infty} \int_{-\pi / 2}^{\pi / 2} d \theta\left[\lambda\left(\frac{B+i \sin \theta}{B-i \sin \theta}\right)^{1 / 2}\right]^{n} T_{n}\left(\frac{\sqrt{B^{2}+1}}{\sqrt{B^{2}+\sin ^{2} \theta}}\right) \\
& \quad=\frac{1}{2} \int_{-\pi / 2}^{\pi / 2} d \theta\left[\frac{(B-i \sin \theta)-\lambda^{2}(B+i \sin \theta)}{(B+i \sin \theta)-2 \lambda\left(B^{2}+1\right)^{1 / 2}+\lambda^{2}(B+i \sin \theta)}+1\right] .
\end{aligned}
$$

This integral can be viewed as a contour integral around the unit circle of a meromorphic function with a single pole inside, whose residue can be computed explicitly. A straightforward calculation then yields the value $\pi(1-\lambda)^{-1}$, which implies that

$$
\int_{-\omega / \alpha}^{\omega / \alpha} d u\left(\frac{\cosh p l / 2+i u \sinh p l / 2)}{\cosh p l / 2-i u \sinh p l / 2}\right)^{n} T_{2 n}\left(\frac{\cosh b / 2}{\cosh d / 2}\right)=\pi
$$

for all $n$ half integers. From this the value of $I_{n}(t)$ follows

$$
I^{n}(t)=\sum_{\gamma \text { prim. }} \sum_{p=1}^{\infty} v(\gamma)^{2 n} \frac{l}{\sinh p l / 2} \frac{e^{-t / 4}}{4 \sqrt{\pi} \sqrt{t}} e^{-p^{2} l^{2} / 4 t} .
$$

Computation of $I_{e}^{n}(t)$. For $I_{e}^{n}(t)$ we need only deal with the case $d\left(z, z^{\prime}\right)=0$, in which the expression $E_{2 n}(b, d)$ reduces to $T_{2 n}(\cosh b / 2)=\cosh b n$, by the defining property of Chebyshev polynomials. The difference $g_{n}^{t}(z, z)-g_{n-[n]}^{t}(z, z)$ only involves elementary integrals, and works out to be

$$
g_{n}^{t}(z, z)-g_{n-[n]}^{t}(z, z)=2 \sum_{0 \leqq m<n-\frac{1}{2}} e^{(n-m)(n-m-1) t} \frac{2 n-2 m-1}{4 \pi} .
$$

As a consequence

$$
\begin{aligned}
I_{e}^{n}(t)= & -2 \chi(M) \sum_{0 \leqq m<n-\frac{1}{2}}(2 n-2 m-1) e^{(n-m)(n-m-1) t} \\
& -4 \pi \chi(M) \frac{e^{-t / 4}}{(4 \pi t)^{3 / 2}} \int_{0}^{\infty} d b \frac{b e^{-b^{2} / 4 t}}{\sinh b / 2} \cosh (n-[n]) b .
\end{aligned}
$$

Adding (2.7) and (2.9) gives the complete formula for the trace of heat kernels on spinors-tensors of arbitrary weights.

We observe that more general formulas for traces of functions of the Laplacians can be found in Hejhal [5].

Zero Modes of the Laplacians. It will be necessary to determine the number $N_{n}^{ \pm}$of zero modes of the Laplacians $A_{n}^{ \pm}$. Except for $N_{1 / 2}^{-}$and $N_{-1 / 2}^{+}$, they are classically 
known and can be read off from the formula $N_{n}^{ \pm}=\lim _{t \rightarrow \infty} \operatorname{Tr}\left(e^{-t \Delta_{n}^{ \pm}}\right)$ $=\lim _{t \rightarrow+\infty} e^{-t n(n \pm 1)} \operatorname{Tr} e^{+t D_{n}}$. Evidently $N_{n}^{ \pm}=N_{-n}^{\mp}$, so we restrict ourselves to $n \geqq 0$. The asymptotic behavior of the traces as $t \rightarrow+\infty$ can then be obtained from (2.7) and (2.9):

$$
\begin{gathered}
\operatorname{Tr}\left(e^{-t \Delta \frac{ \pm}{0}}\right) \sim I^{0}(t) \\
\operatorname{Tr}\left(e^{-t \Delta_{1} / 2}\right) \sim e^{t / 4}\left(I^{1 / 2}(t)+o\left(e^{-t / 4}\right)\right) \\
\operatorname{Tr}\left(e^{-t \Delta_{1 / 2}^{+}}\right) \sim e^{-3 t / 4}\left(I^{1 / 2}(t)+o\left(e^{-t / 4}\right)\right) \\
\operatorname{Tr}\left(e^{-t \Delta_{n}^{ \pm}}\right) \sim e^{-\operatorname{tn}(n \pm 1)} I^{n-[n]}(t)-(2 n-1) \chi(M) e^{(-n \mp n) t},
\end{gathered}
$$

Now $\Delta_{0}^{ \pm}$is the usual Laplacian on functions, which has exactly one zero mode, so that $I^{0}(t) \rightarrow 1$ as $t \rightarrow \infty$. From (2.11) and the fact that $\operatorname{Tr}\left(e^{-t \Delta_{1 / 2}}\right)$ must remain bounded for $t$ large, we deduce that $\left|I^{1 / 2}(t)\right| \leqq C e^{-t / 4}$. It follows then from (2.12) and (2.13) that

$$
\begin{aligned}
& N_{0}^{ \pm}=1, \\
& N_{n}^{+}=0 \quad \text { for all } n \geqq \frac{1}{2}, n \text { half integer, } \\
& N_{1}^{-}=1-\chi(M), \\
& N_{n}^{-}=-(2 n-1) \chi(M) \text { for } n \geqq 3 / 2, n \text { half integer. }
\end{aligned}
$$

Finally we note that $N_{1 / 2}^{-}=N_{-1 / 2}^{+}$corresponds to the number of zero modes of the Dirac operator. It is known (see Hitchin [8]) that these depend on the spin structure on $M$ for $h \leqq 2$, while for $h \geqq 3$ they even depend on the conformal class of the metric. No simple formula such as (2.14) can therefore exist.

\section{Calculation of Determinants}

We shall evaluate determinants by the zeta function method. Recall that

$$
\operatorname{det}^{\prime} \Delta_{n}^{ \pm}=\exp \left(-\left.\frac{d}{d s}\right|_{s=0} \zeta_{n}^{ \pm}(s)\right)
$$

where

$$
\zeta_{n}^{ \pm}(s)=\operatorname{Tr}^{\prime}\left(\Delta_{n}^{ \pm}\right)=\frac{1}{\Gamma(s)} \int_{0}^{\infty} d t t^{s-1}\left[\operatorname{Tr}\left(e^{-t \Delta_{n}^{ \pm}}\right)-N_{n}^{ \pm}\right]
$$

and ' denotes deletion of zero modes whenever they. exist. Since $\operatorname{det}^{\prime} \Delta_{-}^{ \pm}{ }_{n}=\operatorname{det}^{\prime} \Delta_{n}^{\mp}$, we consider only the case $n \geqq 0$. As indicated by (2.14), we discuss separately the cases of $\Delta_{n}^{+}, n \geqq \frac{1}{2}, \Delta_{n}^{-}$for $n \geqq \frac{3}{2}$, and $\Delta_{1}^{-}$. Substituting (2.7), (2.9), and (2.14) into (3.2) yields

$$
\zeta_{n}^{+}(s)=\zeta_{n, e}^{+}(s)+\frac{1}{\Gamma(s)} \sum_{\gamma \text { prim. }} \sum_{p=1}^{\infty}(v(\gamma))^{2 n} \frac{l}{\sinh p l / 2} \cdot \frac{1}{\sqrt{\pi}}\left(\frac{p l}{2 n+1}\right)^{s-\frac{1}{2}} \cdot K_{s-\frac{1}{2}}\left(\left(n+\frac{1}{2}\right) p l\right)
$$


with

$$
\zeta_{n, e}^{+}(s)=\frac{1}{\Gamma(s)} \int_{0}^{\infty} d t t^{s-1} e^{-t n(n+1)} I_{e}^{n}(t)
$$

All integrals and series converge. It follows that

$$
\log \operatorname{det} \Delta_{n}^{+}=-\zeta_{n, e}^{+\prime}(0)-\sum_{\gamma \text { prim. }} \sum_{p=1}^{\infty} v(\gamma)^{2 n} \frac{1}{p\left(1-e^{-p l}\right)} e^{-(n+1) p l} .
$$

We introduce the two Selberg zeta functions

$$
Z_{n}(s)=\prod_{\gamma \text { prim. }} \prod_{p=0}^{\infty}\left(1-v(\gamma)^{2 n} e^{-(p+s) l}\right) \quad \text { for } \quad n=0, \frac{1}{2} .
$$

The analytic continuation of $\zeta_{n, e}^{+}(s)$ to $s=0$ is obtained by performing the $t$-integration first, and by using the small $b$ asymptotic expansion of the integrand to isolate the pieces which are not manifestly convergent but have simple analytic continuations. The result is

$$
\zeta_{n, e}^{+\prime}(0)=c_{n} \chi(M)
$$

with

$$
\begin{aligned}
c_{n}= & \sum_{0 \leqq m<n-\frac{1}{2}}(2 n-2 m-1) \log (2 n-m)-\left(n+\frac{1}{2}\right)^{2}+2(n-[n]) \\
& \cdot\left(n+\frac{1}{2}\right)+\left(n+\frac{1}{2}\right) \log 2 \pi+2 \zeta^{\prime}(-1),
\end{aligned}
$$

where $\zeta(s)$ is the Riemann zeta function. The final formula for $\operatorname{det} \Delta_{n}^{+}$is then

$$
\operatorname{det} \Delta_{n}^{+}=Z_{n-[n]}(n+1) e^{-c_{n} \chi(M)}
$$

for all $n \geqq \frac{1}{2}, n$ half-integer.

We turn next to the case of $\Delta_{n}^{-}$for $n \geqq \frac{3}{2}$. In this case there are zero modes, and we subtract them from the contribution $e^{-n(n-1) t} I_{e}^{n}(t)$ of the identity element to the heat kernel, obtaining

$$
\zeta_{n}^{-}(s)=\zeta_{n, e}^{-}(s)+\frac{1}{\Gamma(s)} \sum_{\gamma \text { prim. }} \sum_{p=1}^{\infty} v(\gamma)^{2 n} \frac{l}{\sinh p l / 2} \frac{1}{\sqrt{\pi}}\left(\frac{p l}{2 n-1}\right)^{s-\frac{1}{2}} K_{s-\frac{1}{2}}\left(\left(n-\frac{1}{2}\right) p l\right),
$$

with

$$
\zeta_{n, e}^{-}(s)=\frac{1}{\Gamma(s)} \int_{0}^{\infty} d t t^{s-1}\left[e^{-t n(n-1)} I_{e}^{n}(t)+2(2 n-1) \chi(M)\right]
$$

This time analytic continuation for $\zeta_{n, e}^{-}$yields $\zeta_{n, e}^{-\prime}(0)=c_{n-1} \chi(M)$ with $c_{n}$ given by (3.5). Thus the formula for $\operatorname{det}^{\prime} \Delta_{n}^{-}$is

$$
\operatorname{det}^{\prime} \Delta_{n}^{-}=Z_{n-[n]}(n) e^{-c_{n-1}} \chi(M)
$$

for all $n \geqq \frac{3}{2}, n$ half-integer.

As for the cases of $\Delta_{0}^{ \pm}$and $\Delta_{1}^{-}$, there is one zero mode, to be subtracted from $I^{0}(t)$. We have to apply then the regularization process of [1(d), Sect. 6]. The 
conclusion is

$$
\begin{gathered}
\zeta_{0}^{ \pm}(s)=\zeta_{0, e}^{ \pm}(s)+\lim _{\delta \rightarrow 0^{+}}\left(\sum_{\gamma \text { prim. }} \sum_{p=1}^{\infty} \frac{l}{\sinh p l / 2} \frac{1}{\Gamma(s)}\right. \\
\left.\cdot \int_{0}^{\infty} d t t^{s-1} e^{-\delta t} \frac{1}{4 \sqrt{\pi} \sqrt{t}} e^{-p^{2} l^{2} / 4 t}-\delta^{-s}\right), \\
\zeta_{0, e}^{ \pm}(s)=\frac{1}{\Gamma(s)} \int_{0}^{\infty} d t t^{s-1} I_{e}^{0}(t), \\
\zeta_{0, e}^{ \pm \prime}(0)=c_{0} \chi(M), \\
\log \operatorname{det}^{\prime} \Delta_{0}^{ \pm}=-c_{0} \chi(M)-\lim _{\delta \rightarrow 0^{+}}\left(\sum_{\gamma \text { prim. }} \sum_{p=1}^{\infty} \frac{1}{p\left(e^{l p}-1\right)} e^{-\delta l p}+\log \delta\right) \\
=-c_{0} \chi(M)+\lim _{\delta \rightarrow 0^{+}} \log \frac{Z_{0}(1+\delta)}{\delta}, \\
\operatorname{det}^{\prime} \Delta_{0}^{ \pm}=Z_{0}^{\prime}(1) e^{-c_{0} \chi(M)} .
\end{gathered}
$$

This formula also appears in [1(d)] and [7]. Similarly

$$
\operatorname{det}^{\prime} \Delta_{1}^{-}=Z_{0}^{\prime}(1) e^{-c_{0} \chi(M)} \text {. }
$$

The only remaining case is $\operatorname{det}^{\prime} \Delta_{1 / 2}^{-}=\operatorname{det}^{\prime} \Delta_{-1 / 2}^{+}$. Although the trace of the heat kernel is available here as in other cases, it is difficult to perform the analytic continuation of $\zeta_{1 / 2}^{-}(s)$ explicitly. The main difficulty comes from the fact that the integrals over $t$ near $\infty$ and sums over $\gamma$ primitive are far from converging absolutely. In addition, the link between $N_{1 / 2}^{-}$and the spin structure as given by the multiplier $v(\gamma)$ is rather subtle. We expect $\operatorname{det}^{\prime} \Delta_{1 / 2}^{-}$to be related to $Z_{1 / 2}\left(\frac{1}{2}\right)$ or $(d / d s)_{\mid s=0}^{N \overline{1} 2} Z_{1 / 2}\left(\frac{1}{2}\right)$, depending on whether there are zero modes.

Finally we observe that in view of the formulas of Polyakov and Alvarez for the conformal anomaly (e.g., [1(c), formula (4.27)]), determinants of Laplacians for general metrics can be obtained from the ones for constant curvature metrics, up to a factor involving the volume of the space of quadratic differentials.

Acknowledgements. It is a pleasure to thank S. Zelditch and J. Sturm for very helpful discussions.

\section{References}

1. (a) Polyakov, A.M.: Quantum geometry of bosonic strings. Phys. Lett. 103B, 207 (1981); Quantum geometry of fermionic strings. Phys. Lett. 103B, 211 (1981)

(b) Friedan, D.: Introduction to Polyakov's string theory." Les Houches XXXIX (1982) 839, Elsevier Publishing

(c) Alvarez, O.: Theory of strings with boundaries. Nucl. Phys. B 216, 125 (1983)

(d) D'Hoker, E., Phong, D.H.: Multiloop amplitudes for the bosonic Polyakov string. Nucl. Phys. B 269, 205 (1986)

(e) D'Hoker, E., Phong, D.H.: Length-twist parameters in string path integrals. Phys. Rev. Lett. 56, 912 (1986)

2. Fay, J.: Fourier coefficients of the resolvent for a Fuchsian group. J. Reine Angew. Math. 293, $143(1977)$ 
3. (a) Ray, D., Singer, I.M.: Analytic torsion for complex manifolds. Ann. Math. 98, 154 (1973) (b) Donnelly, H.: On the analytic torsion and eta invariants for negatively curved manifolds. Am. J. Math. 101, 1365 (1979)

4. McKean, H.: Selberg's trace formula as applied to a compact Riemann surface. Commun. Pure Appl. Math. 25, 223 (1972)

5. Hejhal, D.: The Selberg trace formula for PSL (2, R). Lecture Notes on Mathematics, Vol. 548. Berlin, Heidelberg, New York: Springer 1976

6. Mandelstam, S.: Determinants of Laplacians of string diagrams. Seminar talk at Workshop on Unified String Theories, Santa Barbara, 1985

7. Fried, D.: Analytic torsion and closed geodesics on hyperbolic manifolds (to appear)

8. Hitchin, N.: Harmonic spinors. Adv. Math. 14, 1 (1974)

Communicated by A. Jaffe

Received December 10, 1985; in revised form January 22, 1986 
